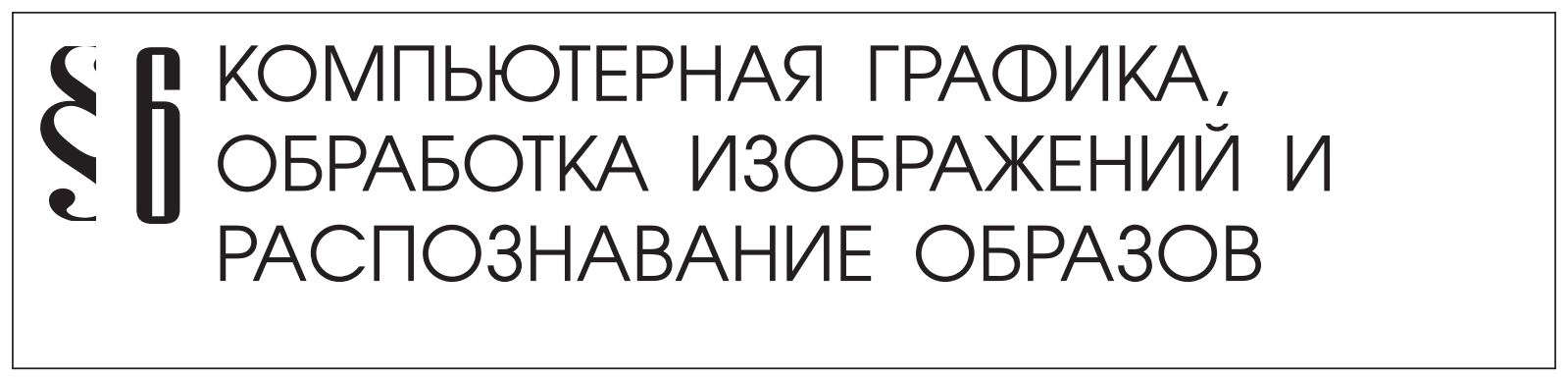

Уржумов Д.В., Кревецкий А.В.

\title{
ИССЛЕДОВАНИЕ ДОСТАТОЧНОЙ СТАТИСТИКИ РАЗЛИЧЕНИЯ ГРУППОВЫХ ТОЧЕЧНЫХ ОБЪЕКТОВ С ЦЕПОЧЕЧНОЙ И ОБЛАЧНОЙ СТРУКТУРАМИ ПО ФОРМЕ ИХ ГРАФОВ ИЕРАРХИЧЕСКОЙ ГРУППИРОВКИ
}

\begin{abstract}
Аннотация: Выполнена параметризация моделей локационных изображений групп точечных объектов типов «цепочка» и «скопление». Исследованы вероятностные характеристики достаточной статистики их различения, необходимые для выбора решающих правил, оптимальных по заданным критериям в различных условиях наблюдения. Рассмотрена методика моделирования наблюдаемых искажений эталонных цепочек, которая все многообразие условий наблюдения позволяют свести к двум параметрам кривизне траектории цепочки и уровню отклонений наблюдаемых координат точечных объектов от их эталонных положений. Такая методика позволяет формализовать и снизить трудоемкость сопоставления конкурирующих методов опознавания цепочек. Рассмотрены особенности программного комплекса для тестирования конкурирующих алгоритмов различения групповых объектов. В качестве статистики различения предложено отношение диаметра графа иерархической группировки обнаруженныхобъектов к суммарной длине ребер этого графа. На основеданных математических моделей методом статистических испытаний получены выборочные оценки законов распределения вероятностей достаточной статистики различения для различных значений параметров моделей. Свойства рассмотренного метода различения чепочек и скоплений с учетом меньшей его трудоемкости делают челесообразным его использование при построении систем распознавания групповых точечных объектов в условиях высокой априорной неопределенности относительно параметров условий наблюдения при мощности групп не менее 10. Предложенная архитектура программного комплекса позволяет тестировать алгоритмы распознавания с различной по числу и типу параметров сигнатурой.. Ключевые слова: различение, опознавание чепочек, групповой точечный объект, распознавание группы объектов, нестационарная форма, контурный анализ, распознавание по форме, анализ формы графа, граф иерархической группировки, структурный анализ графа
\end{abstract}




\section{Введение}

Объекты искусственного происхождения, расположенные в районе проведения дистанционного зондирования земли (ДЗ3), часто имеют размеры сопоставимые с разрешением датчика изображений. При дешифрировании ДДЗ такие объекты интерпретируют как точечные объекты (ТО). В связи с отсутствием признака формы у изображений точечных объектов их идентификация с высоким уровнем достоверности обычно возможна, когда анализируются достаточно устойчивые признаки взаимного расположения соседних точечных объектов. Для реализации такого подхода необходимо вначале обнаружить и распознать такие группы точечных объектов (ГТО) Для снижения числа проверяемых гипотез при решении задач распознавания ГТО проводят предварительное различение ГТО с цепочечной и облачной структурами [1, 2]. Такое разветвление комплексного алгоритма распознавания ГТО позволяет также использовать на последующих этапах различные наборы информативных признаков и методов распознавания.

В предыдущем исследовании [2] был предложен алгоритм опознавания цепочек ТО по их графу иерархической группировки, обеспечивающий теоретически повышенную устойчивость к кривизне траектории расположения объектов цепочки по сравнению с применяемым в настоящее время методом анализа автокорреляционной функции (АКФ) ассоциированного сплошного образа (АСО) [3].

Цель настоящей работы состоит в параметризации моделей наблюдаемых цепочек и скоплений и исследовании вероятностных характеристик достаточной статистики их различения, необходимых для выбора решающих правил, оптимальных по заданным критериям в различных условиях наблюдения.

\section{Параметризация моделей наблюдаемых цепочек и скоплений и достаточной статистики их различения}

Для целей статьи предполагается, что задачи обнаружения отдельных точечных и малоразмерных объектов на фоне крупноразмерных объектов, а также пространственной локализации ГТО решены. Для опознавания предъявляется множество $V$ из $N$ точечных отметок с координатами $\mathbf{x}_{n}=x_{n}+i \cdot y_{n}$ и яркостями $s_{n}$ :

$$
V=\left\{v_{n}\right\}=\left\{\mathbf{x}_{n}, s_{n}\right\}, n=0,1,2, \ldots, N-1, \quad i=\sqrt{-1} .
$$

Данное множество можно представить в виде изображения

$$
s(\mathbf{x})=s(x+i y)=\sum_{n=0}^{N-1} s_{n} \delta\left(\mathbf{x}-\mathbf{x}_{n}\right),
$$

где $x \in[0 ; X[, y=[0 ; Y[$ - координаты произвольной точки на комплексной плоскости изображения в кадре с размерами $X \times Y, \delta(\cdot)$-символ Кронекера или дельта-функция в зависимости от дискретного или непрерывного случая соответственно.

Для опознавания цепочек яркость уже обнаруженных, т.е. отделенных от фона, точечных объектов не информативна. Поэтому можно принять $S_{n}=1 \forall n$.

Наблюдаемые положения ТО $v_{n}$ вследствие ошибок измерений, естественной ва- 
риабельности формы взаимного положения точек ГТО могут отличаться от некоторых эталонных положений $\mathbf{x}_{\mathrm{p} n}$ на случайное смещение $\xi_{n}=\xi_{x n}+i \xi_{y n}$ :

$$
\mathbf{x}_{n}=\mathbf{x}_{\mathrm{p} n}+\xi_{n} .
$$

Для цепочек положим, что эталонные положения $\mathbf{x}_{\mathrm{p} n}$ - это точки неизвестной гладкой кривой $y=f(x)$, а случайное смещение $\xi_{n}$ подчиняется закону распределения $w(\xi)$.

Наиболее общий подход к обнаружению пространственно компактных ГТО с неизвестной формой описан в монографии [1]. Он базируется на построении графа иерархической группировки наблюдаемого множества точечных объектов по степени пространственной близости и разрушении слишком длинных в статистическом смысле ребер. В частном случае эта мера пространственной близости соответствует евклидовому расстоянию между ТО, а граф иерархической группировки, связывающий ГТО, может быть представлен в виде минимального остовного дерева [1]:

$$
G(V, E), \quad E=\left\{e_{m}\right\}_{m=1, N-1}, e_{m}=\left(v_{k}, v_{l}\right), \quad k, l \in[0, N-1], k \neq l
$$

где $V$ - множество вершин, $E$ - множество ребер графа. В этом случае будем считать, что граф $G(V, E)$ уже сформирован.

У идеальной цепочки $G(V, E)$ степени вершин $\operatorname{dg} v_{0}=\operatorname{dg} v_{N-1}=1$, а $\operatorname{dg} v_{1}=\operatorname{dg} v_{2}=\ldots=\operatorname{dg} v_{N-2}=2$. Для наблюдаемых цепочек степени вершин могут быть больше 2. Наличие таких вершин определяет существование нескольких маршрутов $M_{j}=\left\{v_{0 j}, e_{1 j}, v_{1 j}, e_{2 j}, \ldots, v_{N j-1}\right\}$ с концевыми вершинами $v_{0 j}$ и $v_{N j-1}$ с единичной степенью (рис. 2). Причиной этого являются искажения, связанные как с нарушением последовательностиТО, таки сналичиемшумовых сигналов, неверно интерпретированных как объекты. Множество $E_{0}$ ребер, входящих в самый длинный маршрут с $\left|M_{0}\right|=\operatorname{diam} G(V, E)$, назовем основными. Множество остальных ребер $E_{1} \notin M_{0}$ назовем побочными. На рис. 1 побочные ребра показаны пунктиром.

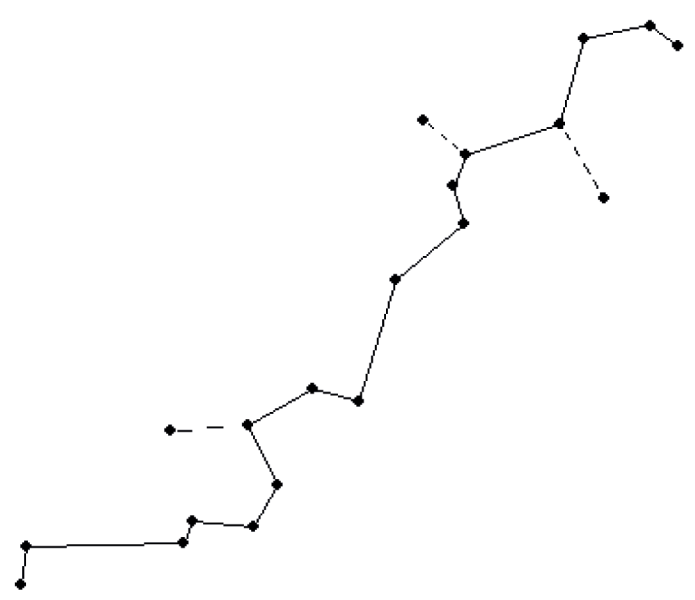

Рис. 1. Пример минимального дерева зашумленной цепочки 
Увеличение доли побочных ребер нарушает структуру цепочки, приближая ее к структуре скопления. С учетом этого в качестве меры непохожести графа $G(V, E)$ на граф ГТО с идеальной цепочечной структурой примем отношение диаметра графа ГТО к суммарной длине ребер:

$$
\mu(G)=\sum_{e_{m} \in E_{0}}\left|e_{m}\right| / \sum_{e_{m} \in E}\left|e_{m}\right|=\left|M_{0}\right| / \sum_{e_{m} \in E_{1}}\left|e_{m}\right| .
$$

Для формализации процедуры тестирования алгоритмов опознавания цепочек, не выходя за рамки рассмотренных выше математических моделей ГТО, сведем все многообразие параметров $f(x)$ и $w(\xi)$ к двум - коэффициенту кривизны траектории цепочки $k$ и среднеквадратическому отклонению $\sigma$ закона случайного отклонения точечных объектов от их эталонных положений.

В качестве коэффициента кривизны $k$ часто используют отношение длины элементарного участка кривой $y=f(x)$ к радиусу кривизны. Такой коэффициент инвариантен к масштабу изображения. Кривая с постоянным коэффициентом кривизны представляет собой окружность. В качестве элементарного участка такой кривой примем сектор окружности между смежными точечными объектами цепочки (см. рис. 2). Тогда коэффициент кривизны

$$
k=\frac{L_{\alpha}}{r}=\frac{\alpha \cdot r}{r}=\alpha
$$

однозначно определяется угловым расстоянием $\alpha$ между смежными объектами эталонной цепочки, выраженном в радианах; $\alpha \in] 0 ; 2 \pi[$

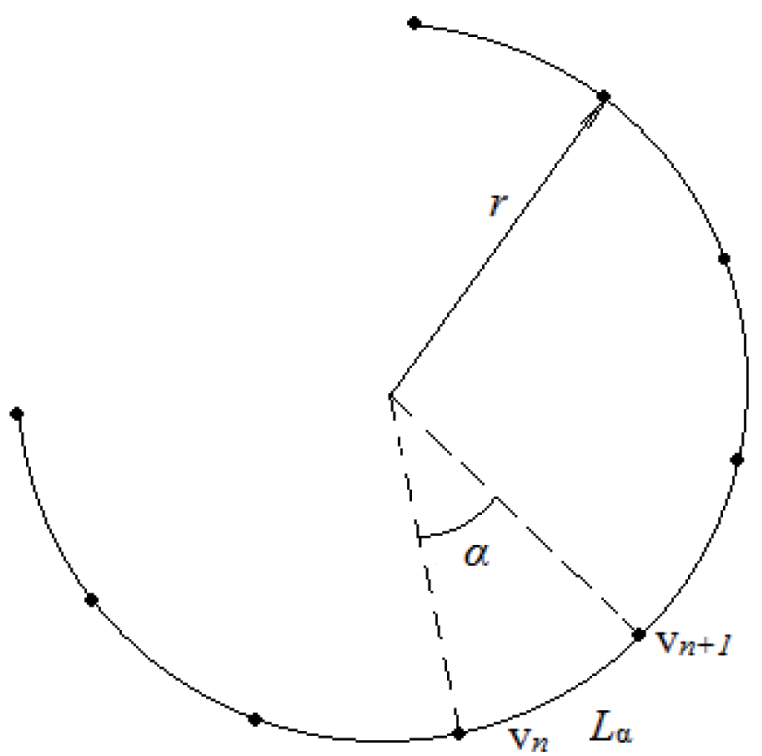

Рис. 2. К определению коэффициента кривизны

В качестве закона распределения независимых шумовых компонент координат то- 
чечных объектов ГТО примем нормальный закон с нулевым математическим ожиданием и среднеквадратическим отклонением $\sigma$ :

$$
w(\xi)=w\left(\xi_{x}+i \xi_{y}\right)=w\left(\xi_{x}\right) w\left(\xi_{y}\right)=\frac{1}{2 \pi \sigma^{2}} \exp \left(-\frac{\xi_{x}^{2}+\xi_{y}^{2}}{2 \sigma^{2}}\right) .
$$

Выбор такого закона распределения обусловлен его симметричностью относительно математического ожидания, одновременным формированием нерегулярности следования точечных объектов как вдоль траектории, так и отклонения вдоль траверза траектории $y=f(x)$, аналитическим видом закона, а также доступностью качественных генераторов таких псевдослучайных чисел в системах моделирования и программирования.

Решение об опознавании ГТО как цепочки принимается при значении отношения (1) меньше порогового $u_{0}$ :

$$
\left\{\begin{array}{l}
H=H_{0}, \text { if } \mu(G) \leq \mu_{0}, \\
H \neq H_{0}, \text { if } \mu(G)>\mu_{0} .
\end{array},\right.
$$

В качестве модели ГТО типа «скопление» примем выборку из случайного равномерного поля $S_{\mathrm{c}}(\mathbf{x})$ точечных отметок (дискретный аналог двумерного пуассоновского потока):

$$
s(\mathbf{x})=\sum_{n} s_{n} \delta\left(\mathbf{x}-\mathbf{x}_{n}\right)=h(\mathbf{x}) s_{\mathrm{c}}(\mathbf{x})
$$

где $h(\mathbf{x})$ - окно выборки.

Распределение длин ребер графа иерархической группировки такого ГТО подчиняется закону Рэлея [3]

$$
W_{\xi}^{(2)}(r)=\frac{d P(\xi<r)}{d r}=\frac{r}{\sigma_{R}^{2}} \exp \left\{\frac{-r^{2}}{2 \sigma_{R}^{2}}\right\}
$$

с параметром $\sigma_{R}^{2}=1 / \pi p$, где $p$-вероятность появления точечного объекта скопления в элементе разрешения кадра наблюдаемого изображения. Для сокращения числа параметров и обеспечения согласованности масштабов моделей цепочек и скоплений будем назначать

$$
p=1 / 2 \bar{r}^{2}
$$

где $\bar{r}=\sqrt{\pi / 2} \cdot \sigma_{R}$-средняя длина ребра графа иерархической группировки. Площадь окна выборки $h(\mathbf{x})$ для формирования скопления определим, исходя из равенства среднего числа N попавших в окно отметок числу точечных объектов цепочки:

$$
E=N / p \text {. }
$$

С целью обеспечения инвариантности рассмотренных моделей к масштабу изображе- 
ния в дальнейшем в качестве уровня координатного шума цепочек будем использовать нормированную величину

$$
a=\sigma / L_{\alpha} .
$$

На рис. 3 изображен пример тестовых сцен с зашумленными цепочками для $k=0,0 ; 3,0 ; 6$, и $a=0,0 ; 0,5 ; 1$.

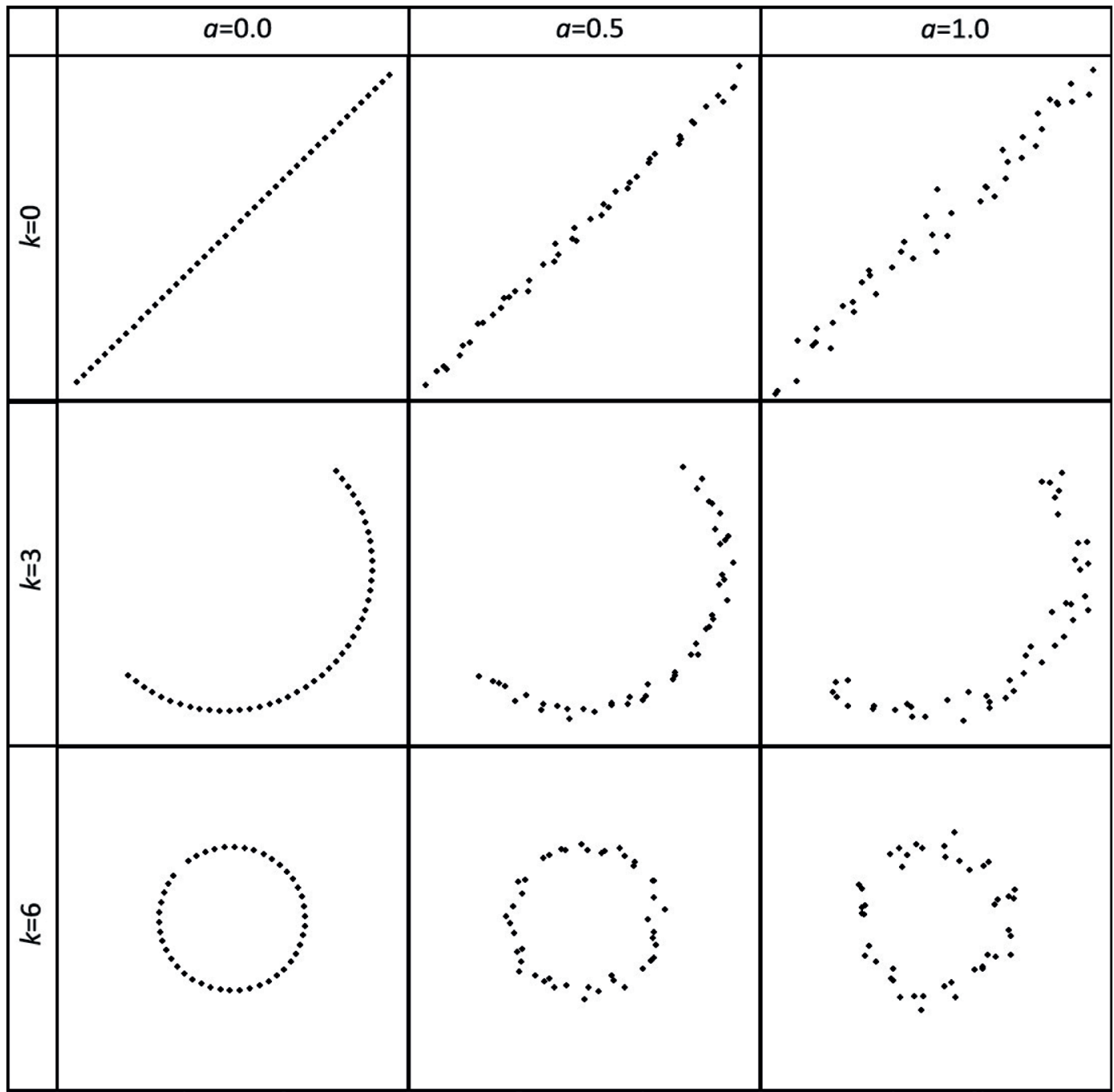

Рис. 3. Примеры зашумленных цепочек

\section{Статистические характеристики достаточной статистики различения цепочек и скоплений}

Для оценки качества различения «цепочки/скопления» и выбора порога принятия решения, оптимального по любой из модификаций байесовского критерия, необходимо получить законы распределения достаточной статистики различения: $v\left(\mu(G) \mid H_{0}, k, a, N\right)$ - условную плотность распределения вероятности статистики различения $\mu$ при наблю- 
дении цепочки и $v\left(\mu(G) \mid H_{1}, k, a, N\right)$ - условную плотность распределения вероятности $\mu$ при наблюдении скопления.

Гистограммы указанных законов достаточной статистики различения приведены на рис. 2,3,4. Сплошной линией (левый график) обозначены гистограммы для скоплений, пунктиром - для цепочек. Каждая из гистограмм получена по 10000 реализациям ГТО, сгенерированных согласно выше описанным параметрическим моделям.

Значения уровня координатных шумов цепочки $a=1,0 ; 0,9 ; 0,8$ выбраны, поскольку графики для ГТО типа цепочка при данном уровне координатного шума расположены ближе всего к графику для ГТО типа скопление.

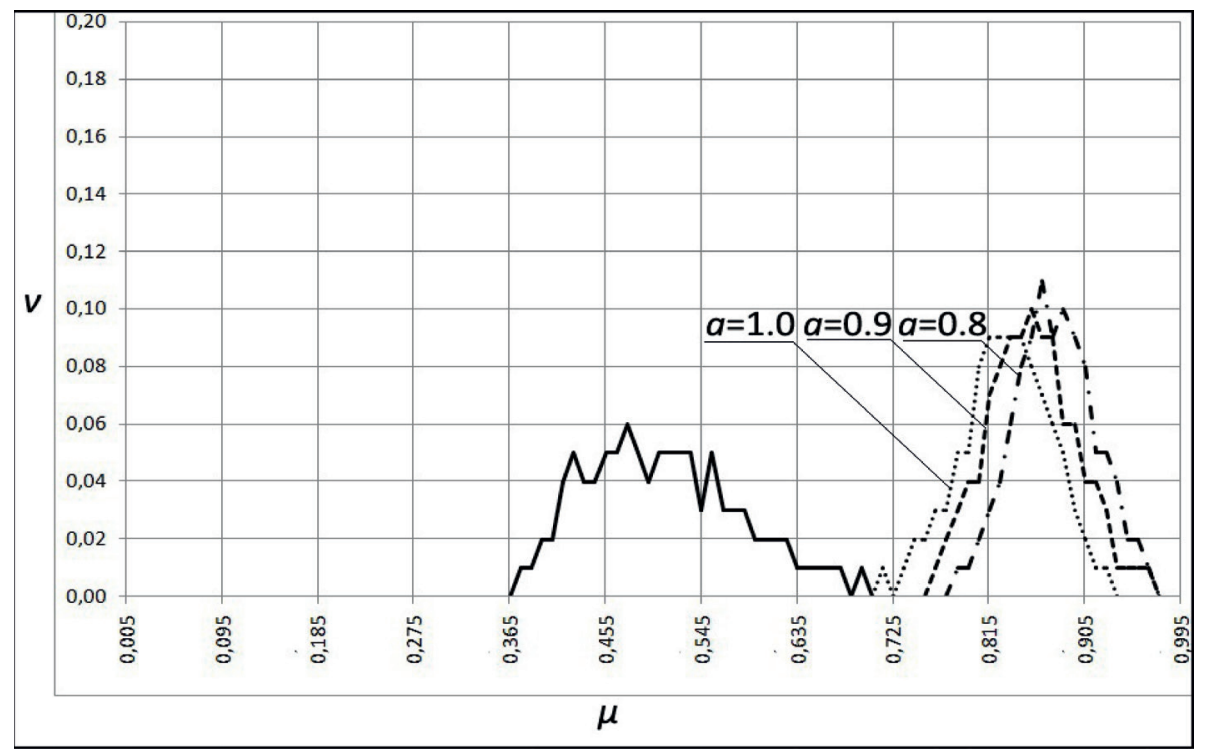

Рис. 4. Гистограммы достаточной статистики различения при $k=0$

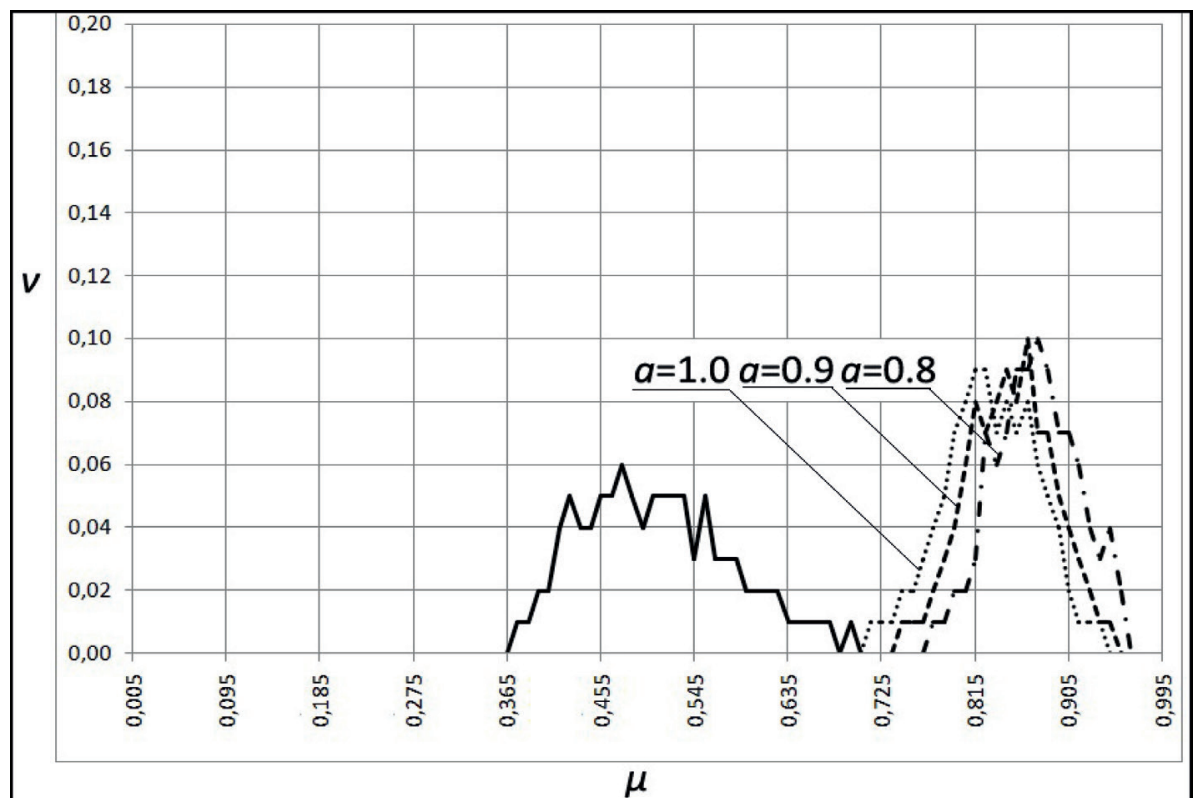

Рис. 5. Гистограммы достаточной статистики различения при $\boldsymbol{k}=3$ 


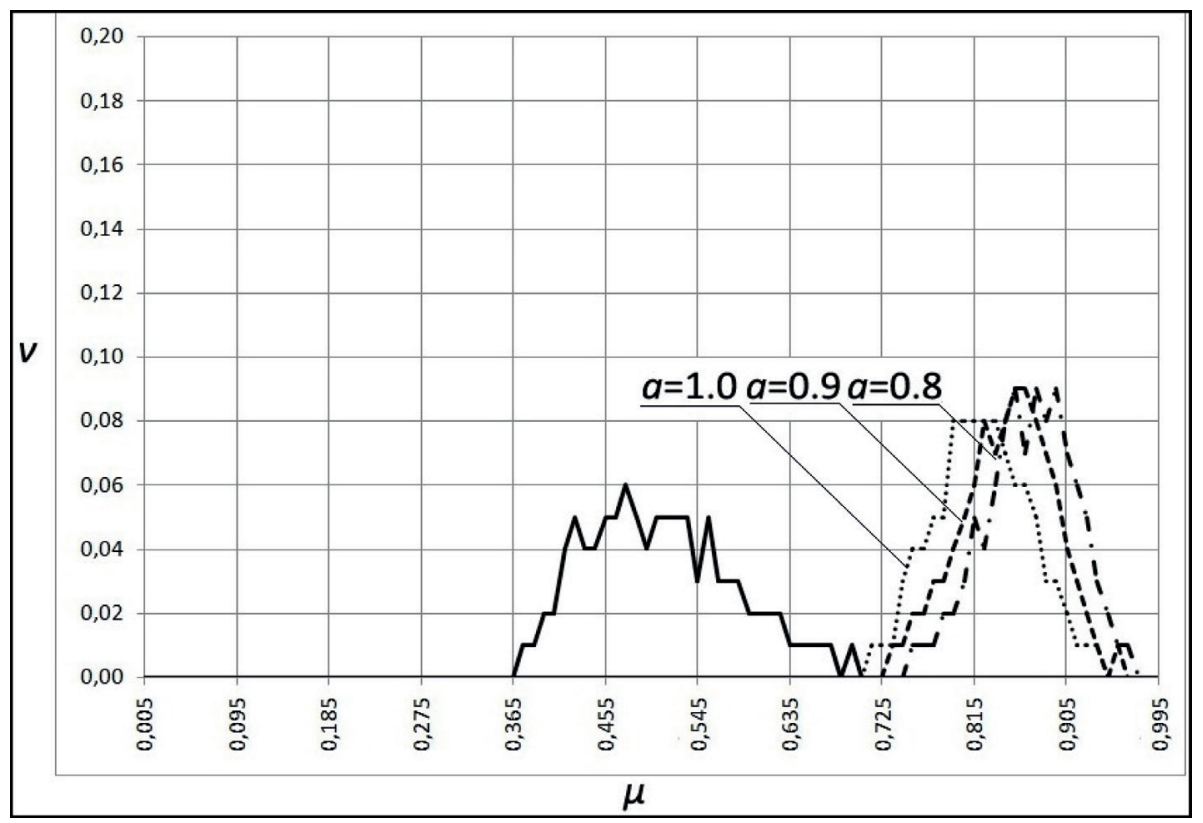

Рис. 6. Гистограммы достаточной статистики различения при $\boldsymbol{k}=6$

Площади под гистограммами правее любого порогового значения $\mu_{0}$ дают оценки условных вероятностей ложного опознавания цепочки (график сплошной линией) и правильного опознавания цепочки (график пунктиром) соответственно. Из полученных графиков в частности видно, что для критерия максимального правдоподобия (минимума суммы условных вероятностей ошибок различения) значение оптимального порога $\mu_{0} \approx 0,7$ и при это вероятность суммарной ошибки близка к нулю. Однако следует отметить, что для ГТО мощностью менее десяти точек условные законы распределения достаточной статистики различения начинают существенно пересекаться. Это объясняется высокой вероятностью отсутствия побочных ребер в графе иерархической группировки скоплений при малом числе точечных объектов.

\section{Особенности архитектуры программного комплекса тестирования алгоритмов распознавания групповых точечных объектов}

На этапе проектирования алгоритмов распознавания и классификации малоразмерных и точечных объектов (МРО и ТО) по данным дистанционного зондирования земли целесообразно проведение исследований их работы методом статистических испытаний на сгенерированных (по имеющимся математическим моделям) наблюдаемых сценах. Сигнатура тестируемых методов может различаться по числу и типу параметров. Таким образом, к программному комплексу тестирования предъявляется требование устойчивой работы с функциями с заранее неопределенной сигнатурой. Это требование не может быть выполнено непосредственно, вследствие отсутствия в современных компилируемых языках программирования методов динамической генерации кода. 
Применение для поставленной задачи интерпретируемых языков нецелесообразно по причине низкой производительности.

Поскольку непосредственно решить задачу динамической генерации программного кода невозможно, необходимо разработать механизм регистрации алгоритма в системе. Программный код алгоритма должен быть скомпилирован отдельно и подключаться по запросу пользователя. Единственной методикой позволяющей реализовать подобное обособленное размещение кода алгоритма является использование динамических библиотек с применением динамической загрузки по запросу пользователя. Поскольку при динамической загрузке библиотеки в основной код дескриптор функции передается в виде указателя на пустой тип void, непосредственное ее использование, а также преобразование в форму допустимую для применения без явного описания сигнатуры функции в коде невозможно. Это обстоятельство явно противоречит требованию к комплексу тестирования и вынуждает перенести подобное преобразование в код динамической библиотеки. Наиболее удобной для реализации во всех последующих алгоритмах является добавление в код библиотеки единственной функции без параметров registrate. Данная функция возвращает класс универсальной спецификации алгоритма, который содержит метод-обертку над функцией, а также упорядоченный список классов, задающих тип и диапазон значений параметров.

Поскольку число параметров различно, то для генерации функций оберток придется создать вложенный класс, представляющий собой библиотеку однотипных статических шаблонных методов, различающихся числом типов:

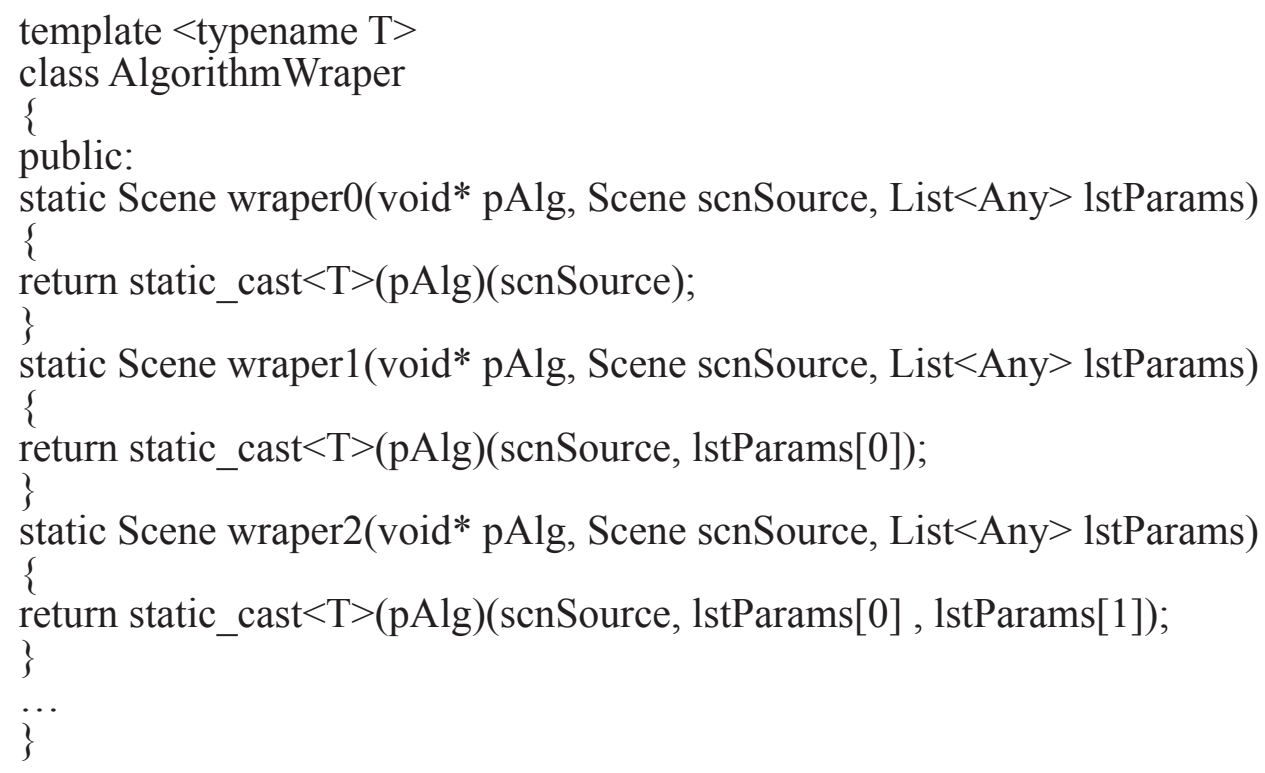

В данном фрагменте кода класс Scene - аналитическое описание сцены; $T$ - шаблонный класс, использование в коде которого, налагает требование указателя на функцию, принимающее соответствующее число аргументов; List $<$ Any $>$ - список контейнеров произвольного типа, void * - указатель произвольного типа. Таким образом, функцию-обертку 
можно хранить в классе-спецификации в виде указателя: Scene (*m_pAlg)(void*, Scene, List $<$ Any>), формат которого един для методов с любым числом и типом параметров.

Вторым компонентом класса-спецификации является класс, хранящий диапазон значений параметра. Диапазон осуществляет взаимодействие с программным интерфейсом элементов управления controlInterface.

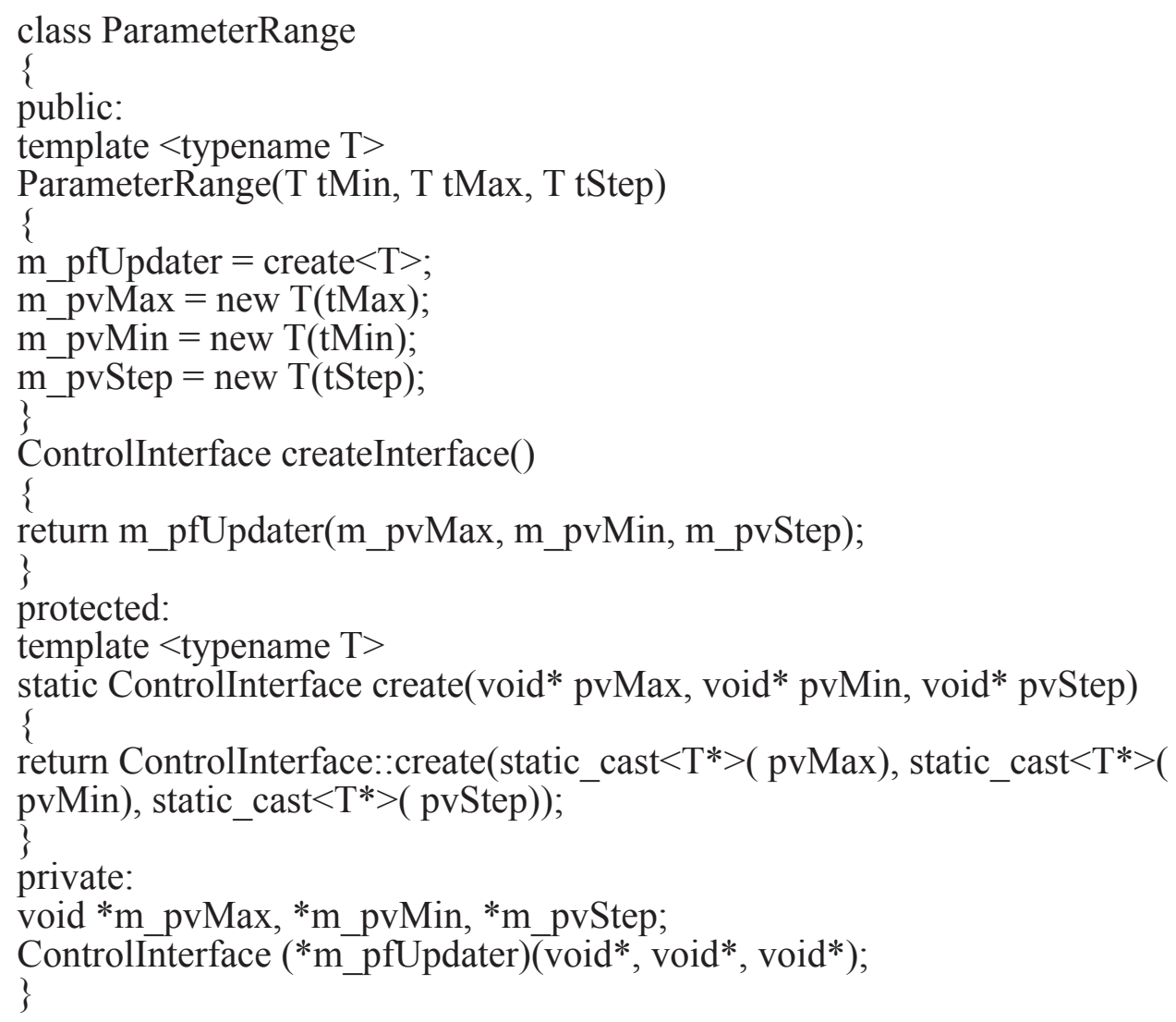

Конструктор класса диапазона принимает три шаблонных параметра: максимальное, минимальное значения и шаг диапазона и осуществляет преобразование их типа к указателю на неспециализированные данные. Обратное преобразование осуществляется при генерации интерфейса элемента управления через функцию, вызываемую по указателю. Такой подход позволяет осуществлять безопасную передачу данных интерфейсу элемента управления без проверки на соответствие типов [4, с. 73].

Приведенного код используется в классе-спецификации алгоритма:

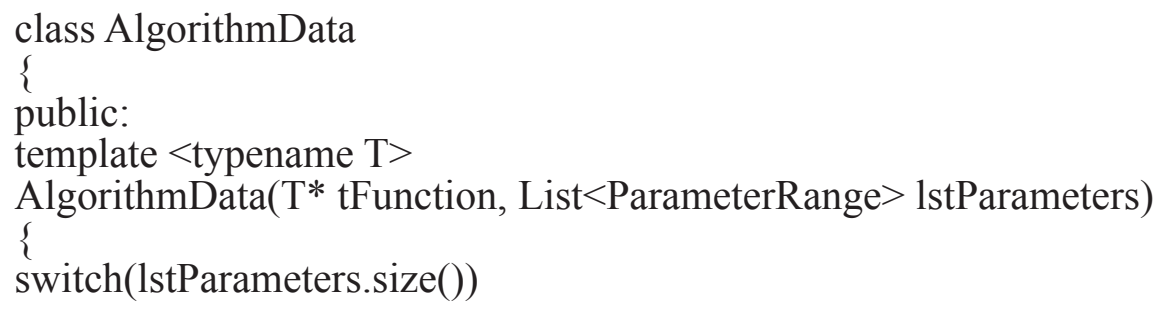




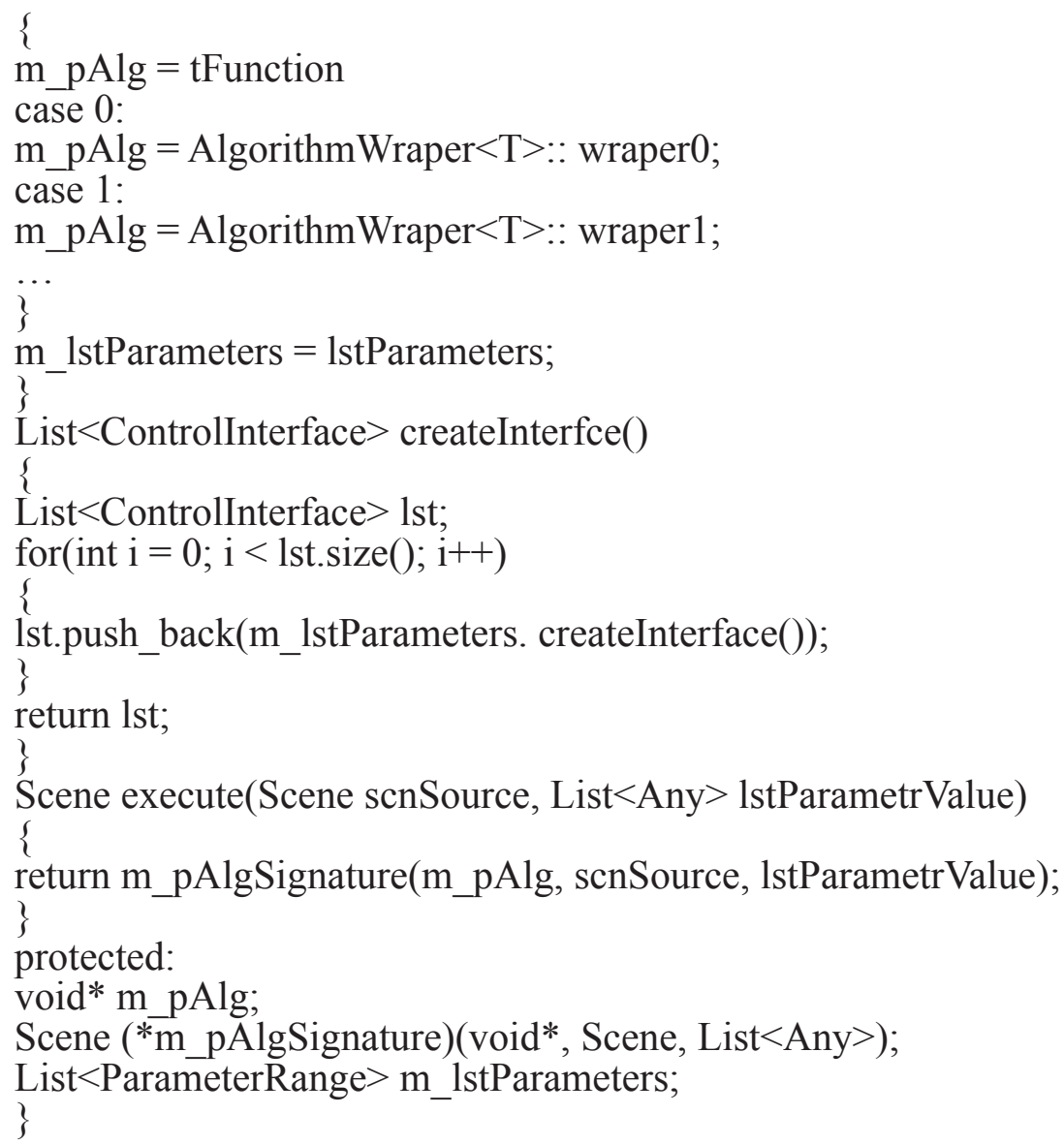

Данный класс преобразует указатель на функцию алгоритма к неспециализированному виду, генерирует и сохраняет функцию-обертку, а так же список классов, хранящих диапазоны параметров. По запросу пользователя класс может предоставить набор интерфейсов для элементов управления, которые позволят пользователю выбрать требуемое значение параметра из диапазона. Получив сигнал от пользовательского интерфейса, содержащий весь список инициализированных пользователем значений параметров, класс запускает алгоритм на выполнение с указанными параметрами.

Таким образом, разработчику кода библиотеки достаточно определить в ней функцию вида:

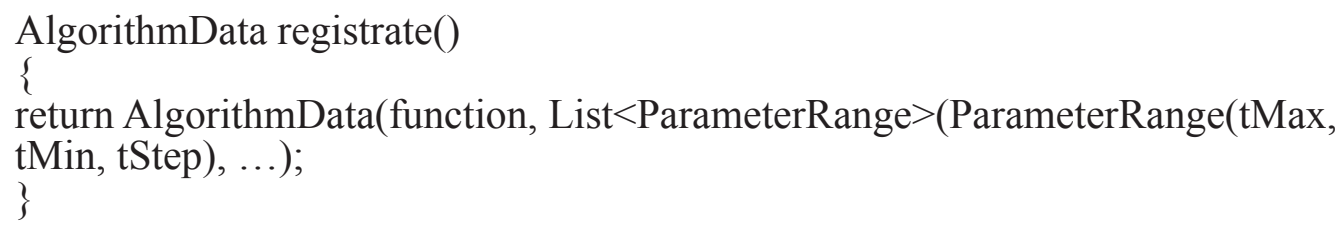

где под function подразумевается имя регистрируемой функции, а List<ParameterRange> - список инициализированных диапазонов параметров.

При загрузке библиотеки комплекс вызывает ее функцию registrate, принимает 
универсальную спецификацию, возвращаемую функцией регистрации. После того как пользователь задаст все параметры, определит набор тестовых сцен и подтвердит начало тестирования, алгоритм будет запущен по указателю на его функцию в универсальном описании. Информация о результатах работы алгоритма сохраняется в дополнительных данных аналитического описания сцены и может быть проверена на соответствие заданным пользователем критериям в автоматическом режиме. Статистика правильных (соответствующих заданному условию) результатов собирается в класс диаграммы и выводится в виде таблицы по завершению нагрузочного тестирования. Отдельно ведется сбор статистики по времени выполнения алгоритма.

Предложенная архитектура позволяет комплексу тестировать алгоритмы с различной по числу и типу параметров сигнатурой. Объем дополнительного кода динамических библиотек алгоритмов, требуемых для обеспечения к ним доступа, минимален. Задачи проверки условия и сбора статистики являются типовыми задачами обработки данных, поэтому в статье лишь сообщается об их интеграции в архитектуру программного комплекса без описания решения.

\section{Заключение}

Предлагаемый в работе метод различения групповых точечных объектов типов «цепочка» и «скопление» по структуре графа иерархической группировки инвариантен к типовым геометрическим преобразованиям наблюдаемого изображения, а также конкретному классу цепочек и скоплений. Сопоставление с используемым в настоящий момент аналогом, где решение выносится по интервалу корреляции контура минимального дерева группового объекта, показывает, что предлагаемый метод обеспечивает более высокие значения вероятности правильного опознавания цепочек при случайных отклонениях точечных объектов от гладкой траектории эталонной цепочки и повышенную устойчивость к изменению кривизны траектории. Перечисленные свойства рассмотренного метода опознавания цепочек с учетом меньшей его трудоемкости делают целесообразным его использование при построении систем распознавания групповых точечных объектов в условиях высокой априорной неопределенности относительно параметров условий наблюдения. Установлено, что указанные преимущества обеспечиваются для групп с достаточно большим числом объектов.

Работа выполнена в рамках проекта РФФИ №13-01-00427

\section{Библиография :}

1. Введение в контурный анализ; приложения к обработке изображений и сигналов / Я.А. Фурман, А.В. Кревецкий, А.К. Передреев, и др.; под ред. Я.А.Фурмана. - 2-е изд. - М.: ФИЗМАТЛИТ, 2003. - 592 с.

2. Уржумов Д.В., Кревецкий А.В. Опознавание плоских цепочек точечных и малоразмерных объектов по структуреихграфаиерархическойгруппировки//ВестникПГТу.Радиотехническиеиинфокоммуникационные системы. - Йошкар-0ла: ПГТУ, 2014. №1 (20). - С. 54-62. 
3. Плекин В.Я., Кревецкий А.В. Обнаружение групповых точечных объектов с нестационарной конфигурацией // Известия вузов. Радиоэлектроника 1994, Том 37, N3.-C. 8-21.

4. Александреску А. Современное проектирование на С++. - М.: «Вильямс», 2002. - 336 с.

\section{References:}

1. Vvedenie v konturnyi analiz; prilozheniya k obrabotke izobrazhenii i signalov / Ya.A. Furman, A.V. Krevetskii, A.K. Peredreev, i dr.; pod red. Ya.A.Furmana. - 2-e izd. - M.: FIZMATLIT, 2003. - 592 s.

2. Urzhumov D.V., Krevetskii A.V. Opoznavanie ploskikh tsepochek tochechnykh i malorazmernykh ob"ektov po strukture ikh grafa ierarkhicheskoi gruppirovki // Vestnik PGTU. Radiotekhnicheskie i infokommunikatsionnye sistemy. - loshkar-0la: PGTU, 2014. №1 (20). - S. 54-62.

3. Plekin V.Ya., Krevetskii A.V. Obnaruzhenie gruppovykh tochechnykh ob"ektov s nestatsionarnoi konfiguratsiei // Izvestiya vuzov. Radioelektronika 1994, Tom 37, N3.-S. 8-21.

4. Aleksandresku A. Sovremennoe proektirovanie na (++. - M.: «Vil'yams», 2002. - 336 s. 